\title{
TRATAMIENTO DE LIXIVIADOS UTILIZANDO HUMEDALES CONSTRUIDOS Y DETERMINACIÓN DE CONDUCTIVIDADES HIDRÁULICAS EN CLIMA TROPICAL
}

\section{TREATMENT OF LEACHATE USING CONSTRUCTED WETLANDS AND DETERMINATION OF HYDRAULIC CONDUCTIVITIES IN A TROPICAL CLIMATE}

\author{
Yina F. Jiménez-Cerón ${ }^{1}$, Laura I. Delgado-Calvache ${ }^{2}$, Carolina Fernández-Tulande ${ }^{3}$, Huber M. Pino-Alegría ${ }^{4}$, \\ Juan C. Casas-Zapata ${ }^{5}$, Carlos A. Madera-Parra ${ }^{6}$, Jaime A. Lara-Borrero ${ }^{7}$, Jordi Morató-Farreras ${ }^{8}$, Edwin Rengifo-Canizales ${ }^{9}$
}

${ }^{1}$ Ing. Ambiental. Universidad del Cauca, Grupo de investigación Ciencia e Ingeniería en Sistemas Ambientales-GCISA, Calle 5 No- 4-70, Popayán-Cauca, Colombia, e-mail: yfceron@unicauca.edu.co, (iDhttps://orcid.org/0000-0002-4324-2531; ${ }^{2}$ Ing. Ambiental. Universidad del Cauca. GCISA, Calle 5 No. 4-70, Popayán-Cauca, Colombia, e-mail: laura.delgado.1512@gmail. com; ${ }^{3}$ Ing. Ambiental. Universidad del Cauca. GCISA, Calle 5 No. 4-70, Popayán-Cauca, Colombia, e-mail: carofernandezt@ gmail.com, (Dhttps://orcid.org/0000-0002-4208-0251; ${ }^{4}$ Universidad del Cauca. GCISA, Calle 5 No 4-70, Popayán-Cauca, Colombia, e-mail: manuelpino@unicauca.edu.co, @iDhttps://orcid.org/0000-0002-8539-914X; ${ }^{5}$ Ph.D. en Ingeniería, Profesor Titular. Universidad del Cauca, Facultad de Ingeniería Civil, Departamento de Ingeniería Ambiental y Sanitaria, Calle 5 No. 4-70, Popayán-Cauca, Colombia, e-mail: jccasas@unicauca.edu.co, (DiDttps://orcid.org/0000-0003-3796-237X; ${ }^{6} \mathrm{Ph} . D$ en Ingeniería, Profesor Titular. Universidad del Valle, Escuela EIDENAR, Facultad de Ingeniería, Calle 13 No. 100-00, Cali-Valle, Colombia, e-mail: carlos.a.madera@correounivalle.edu.co, (iDhttps://orcid.org/0000-0002-9147-9795; ${ }^{7}$ Ph.D. en Territorio y Medio Ambiente, Profesor Titular. Pontificia Universidad Javeriana, Departamento de Ingeniería Civil, Carrera 7 No. $40-62$, Bogotá, D.C., Colombia, e-mail: laraj@javeriana.edu.co, Đibttps://orcid.org/0000-0003-3247-7519; ${ }^{8}$ Ph.D. Microbiología, Profesor Titular. Universidad Politécnica de Cataluña, Cátedra UNESCO de Sostenibilidad, Calle Jordi Girona, 31.08034 Barcelona-Cataluña, España, e-mail: jordi.morato@upc.edu, (iDhttp://orcid.org/0000-0003-2588-8846; ${ }^{9}$ M.Sc. en Ingeniería en Sistemas, Profesor Titular. Universidad del Cauca, Facultad de Ciencias Exactas y de la Educación, Departamento de matemáticas, Carrera 2 No. 3N-111, Popayán-Cauca, Colombia, e-mail: erengifo@unicauca.edu.co, (iDhttps://orcid.org/00000002-9331-7757

Rev. U.D.C.A Act. \& Div. Cient. 21(2): 543-552, Julio-Diciembre, 2018

https://doi.org/10.31910/rudca.v21.n2.2018.979

Artículo de acceso abierto publicado por Revista U.D.C.A Actualidad \& Divulgación Científica bajo una licencia Creative Commons CC BY-NC 4.0

\section{RESUMEN}

Los humedales construidos en los últimos años han sido ampliamente usados en la depuración de una gran variedad de aguas residuales, por ser una alternativa que ofrece múltiples ventajas, en comparación con otros sistemas de tratamiento. Este estudio evaluó la eficiencia de seis sistemas de humedales, construidos de flujo subsuperficial horizontal (HCFSSH), a escala piloto, para la remoción de materia orgánica (DQO), nutrientes $\left(\mathrm{N}_{-} \mathrm{NO}_{3}{ }^{-}, \mathrm{P}_{-}-\mathrm{PO}_{4}{ }^{3-}\right)$ y plomo $\left(\mathrm{Pb}^{+2}\right)$, además de la incidencia del tiempo de operación sobre la conductividad hidráulica (Ks) para tratar lixiviados del Relleno Sanitario "El Ojito", del municipio de Popayán, Cauca. Tres humedales fueron plantados con Heliconia psittacorum (HCFSSH- $H$. p) y tres con Cyperus haspan (HCFSSH-C. h); el medio de soporte utilizado fue grava, operados con un caudal de $0.012 \mathrm{~m}^{3} /$ día y un tiempo de retención hidráulico de 4 días; las unidades recibieron efluente de una laguna de oxidación. Los resultados mostraron remociones medias de: DQO (51,67 y $64,00 \%), \mathrm{N}^{-N^{3-}}(45,33$ y $48,17 \%), \mathrm{P}^{-P^{3--}}(53,67$ y $62,67 \%)$ y $\mathrm{Pb}^{+2}(5,74$ y $14,75 \%)$ para los HCFSSH- $H$. $p$ y HCFSSH-C. $h$, respectivamente y valores de conductividad hidráulica, entre 700 y $1000 \mathrm{~m} /$ día. El sistema de humedales mostró ser eficiente para el tratamiento secundario de lixiviado de rellenos sanitarios maduros, obteniendo eficiencias de remoción, significativamente mayores, en los HCFSSH-C. $h$.

Palabras clave: materia orgánica, nutrientes, plomo, Heliconia psittacorum, Cyperus haspan. CAB Thesaurus. 


\section{ABSTRACT}

In recent years, constructed wetlands have been widely used for the purification of wastewater, representing an alternative method that offers multiple advantages when compared to other treatment systems. The present study assessed the efficiency of six horizontal subsurface flow constructed wetlands (HSSFCW) at pilot scale for the removal of organic matter (COD), nutrients (N-NO $\left.{ }_{3}^{-}, \mathrm{P}_{-} \mathrm{PO}_{4}{ }^{3-}\right)$ and lead $\left(\mathrm{Pb}^{2+}\right)$. The study also looked at the operating time on hydraulic conductivity (Ks) to treat leachate from the "El Ojito" Sanitary Landfill in the municipality of Popayán, Cauca. Three wetlands were planted with Heliconia psittacorum (HSSFCW-H. $p$ ), and three with Cyperus haspan (HSSFCW-C. $h$ ). The support medium used was gravel. They were operated at a flow of $0.012 \mathrm{~m}^{3} /$ day, and a hydraulic retention time of 4 days. The units received effluent from the oxidation pond. The results showed mean removals of COD (51.67 and 64.00\%), $\mathrm{N}-\mathrm{NO}_{3}{ }^{-}(45.33$ and $48.17 \%), \mathrm{P}_{-} \mathrm{PO}_{4}{ }^{3-}(53.67$ and $62.67 \%)$ and $\mathrm{Pb}^{+2}$ (5.74 and $14.75 \%$ ) for HSSFCW-H. $p$ and HSSFCW-C. $h$ respectively. Hydraulic conductivity values were between 700 and $1000 \mathrm{~m} /$ day. The wetland system proved to be efficient for the secondary treatment of leachate from mature landfills, obtaining removal efficiencies significantly higher in HSSFCW-C. $h$.

Keywords: organic matter, nutrients, lead, Heliconia psittacorum, Cyperus haspan.

\section{INTRODUCCIÓN}

La disposición y el manejo de residuos sólidos, a nivel mundial, es un tema que demanda cada día un mayor interés, debido a la problemática ambiental, económica y social, que se genera alrededor de estas actividades (Renou et al. 2008). Los rellenos sanitarios continúan siendo ampliamente aceptados y utilizados, por ser un método de bajo costo, para la eliminación de materiales de desecho (Wallace et al. 2015); sin embargo, los lixiviados que se generan tienen propiedades tóxicas y pueden causar efectos nocivos en los ecosistemas, debido a su composición en metales, materia orgánica, productos químicos clorados y altos niveles de nutrientes (Speer et al. 2012).

Se han aplicado diversas tecnologías para la depuración de lixiviados, como tratamientos de tipo convencional, mediante procesos anaerobios, aerobios y sistemas de membrana, aunque, debido a la variabilidad en la calidad y la cantidad de este subproducto, estos sistemas pueden resultar poco eficientes, además de ser costosos, en su etapa constructiva y operativa (Yalcuk \& Ugurlu, 2009). Los humedales construidos, se utilizan en todo el mundo como una tecnología en el tratamiento de aguas residuales, por su buen desempeño, simplicidad y bajo requerimiento de energía (Vymazal, 2014; Yalcuk \& Ugurlu, 2009).

Existen varios tipos de humedales construidos desarrollados con base en las características hidráulicas, nivel de agua y dirección de flujo. Los humedales de flujo subsuperficial son los más aplicados (Fonder \& Headley, 2013) y se han reportado para el tratamiento de lixiviados, remociones en rangos de $26,7-98 \%, 11,1-94 \%$ y $17,3-88 \%$, para DBO, DQO y SST, respectivamente (Chiemchaisri et al. 2009; Yalcuk \& Ugurlu, 2009; Wojciechowska et al. 2010).

En este tipo de tratamiento, se presenta la interacción de varios procesos biológicos, físicos y químicos, que dependen del tiempo de contacto entre las aguas residuales con el medio poroso, biopelícula y las raíces de las plantas (Kadlec \& Wallace, 2009; Knowles et al. 2011; Nivala et al. 2012). Un parámetro importante en los humedales construidos es la conductividad hidráulica, la cual, se ve afectada por el crecimiento de la biopelícula, la deposición de precipitados químicos, la acumulación de lodos y biomasa de la planta bajo el suelo, que disminuyen la cantidad y el tamaño de los espacios vacíos del medio filtrante; una menor conductividad hidráulica se asocia, normalmente, a una menor eficiencia de tratamiento (Pedescoll et al. 2009).

Este estudio tuvo como objetivo determinar la eficiencia de seis sistemas de HCFSSH a escala piloto, plantados con H. psittacorum y C. haspan, en términos de $\mathrm{DQO}, \mathrm{N}-\mathrm{NO}_{3}{ }^{-}$, $\mathrm{P}_{-} \mathrm{PO}_{4}{ }^{3-}, \mathrm{Pb}^{+2}$, además de evaluar la incidencia del tiempo de operación sobre la conductividad hidráulica, tratando lixiviados del Relleno Sanitario "El Ojito", del municipio de Popayán-Cauca.

\section{MATERIALES Y MÉTODOS}

El Relleno Sanitario "El Ojito", se encuentra ubicado en la Vereda Cajete, a $3 \mathrm{~km}$ de la ciudad de Popayán, Colombia ( $\left.2^{\circ} 27^{\prime} 52.5^{\prime \prime} \mathrm{N}, 76^{\circ} 39^{\prime} 10.2^{\prime \prime} \mathrm{W}\right)$, clausurado desde septiembre del 2014, donde se depositaron en promedio $210 \mathrm{t}$ de residuos sólidos por día, generando entre 0,001 y $0,003 \mathrm{~m}^{3} / \mathrm{s}$ de lixiviado.

Se emplearon $6 \mathrm{HCFSSH}$ a escala piloto, en fibra de vidrio, de dimensiones 0,6m de ancho, $1 \mathrm{~m}$ de largo, 0,6m de altura, con tubería de entrada y salida en cada sistema, operados en flujo continuo y una pendiente del $1 \%$. Se instalaron dos piezómetros por cada humedal, a $15 \mathrm{~cm}$ de la entrada (P1) y salida (P2), en tubería de PVC de 2 pulgadas de diámetro y una flauta para distribuir el flujo en la salida. Se utilizó, como medio de soporte, grava lavada; un estudio de granulometría de una muestra representativa indicó un 55\% de tamaño de 1 pulgada y porosidad teórica del $40 \%$, se depositó en las 
unidades experimentales, hasta una altura de 0,30m y una lámina de agua de $0,20 \mathrm{~m}$.

Los humedales, se alimentaron del lixiviado de la laguna de estabilización anaerobia, con tres tanques plásticos de $0,03 \mathrm{~m}^{3}$; cada uno suministró lixiviado a dos humedales, por medio de tubería de 1 pulgada y llaves de cierre lento, manteniendo un caudal de entrada de $0,012 \mathrm{~m}^{3} /$ día y un tiempo de retención hidráulico de 4 días (Ecuación 1), que permite generar los procesos de nitrificación y degradación de la materia orgánica (Crites \& Tchobanoglous, 1998).

$$
\mathrm{Q}=\frac{\mathrm{V}}{\mathrm{t}}=\frac{\varepsilon(\mathrm{LWh})}{\mathrm{t}} \quad \text { Ecuación (1) }
$$

Dónde:

$Q=$ Caudal $m^{3}$

$V=$ Volumen útil $\left(\mathrm{m}^{3}\right)$

$T=$ Tiempo de retención hidráulico (días)

$\varepsilon=$ Porosidad del medio filtrante (es un porciento y se expre-

sa en forma decimal)

$L=$ Longitud del humedal $(\mathrm{m})$

$H=$ Profundidad del agua en el humedal (m)

$$
Q=\frac{0.4 \times(1 \mathrm{~m} \times 0.60 \mathrm{~m} \times 0.20 \mathrm{~m})}{4 \text { días }}=0.012 \frac{\mathrm{m}^{3}}{\text { día }}
$$

La aclimatación de las unidades, se realizó en dos etapas: un mes en condiciones batch y un mes en condiciones de flujo continuo. El montaje experimental, se muestra en la figura 1.

Vegetación: Las especies empleadas fueron $H$. psittacorum y C. haspan, por su tipicidad en el Departamento del Cauca, capacidad de desarrollarse y adaptarse en medios inundados y presentar buenas eficiencias de remoción en diferentes parámetros; se consideraron estudios previos, como los reportados por Madera (2016) y Akinbile et al. (2012), tratando lixiviados de rellanos sanitarios en HCFSSH.

Los esquejes, se sembraron en bolsas y se mantuvieron en un proceso de aclimatación; se regaron con lixiviado diluido en diferentes porcentajes, por un periodo de un mes; luego, se sembraron en las unidades experimentales con una densidad de 6 plantas por $0,6 \mathrm{~m}^{2}$; los humedales 1 , 3 y 5 , se plantaron con $H$. psittacorum y los humedales 2,4 y 6 , con C. haspan.

Variables respuesta: Se determinó la $\mathrm{DQO}$, plomo $\mathrm{Pb}^{2+}$, nitrógeno, como $\mathrm{N}_{-} \mathrm{NO}_{3}{ }^{-}$y fósforo, como ortofosfatos, en el afluente y efluente de los sistemas HCFSSH- $H$. p y HCFSSH-C. $h$, con una frecuencia de muestreo de quince días, durante cuatro meses, además de los parámetros de campo: $\mathrm{pH}$, temperatura, conductividad eléctrica (CE) y oxígeno disuelto (O.D.).
Se calcularon las variables incluidas en la ecuación de Sanford et al. (1995) (Ecuación 2), para encontrar la Ks en los piezómetros P1 y P2, de cada humedal; se cerró la válvula de alimentación y se abrió totalmente la válvula de drenaje; el nivel del agua se midió con tablas rectangulares de madera, procedimiento que se efectuó cada 30 segundos hasta completar 10 minutos; después cada minuto hasta que el nivel del agua, se estabilizó o el flujo en la salida fuera mínimo; finamente, se midió el volumen drenado.

$V_{d}=V^{*}\left[\frac{1-e^{-t^{*} \frac{3 * h(0,0) * K_{s}^{*} \cos \beta}{\varepsilon^{*} L^{2}} \times\left(1-\frac{V^{*}}{V_{i}}\right)}}{\left.1-\frac{V^{*} *}{V_{i}} * e^{-t^{*} \frac{*^{*} h(0,0) * K_{s}^{*} \cos \beta}{\varepsilon^{*} L^{2}} *\left(1-V^{*}\right.}\right)}\right]$

Ecuación (2)

Donde:

$V_{i}=\varepsilon \omega L h(0,0),\left(\mathrm{m}^{3}\right)$

$V^{*}=\varepsilon \omega L[h(0,0)-h(0, t)]+\frac{1}{2} \varepsilon \omega L^{2} \tan \beta,\left(\mathrm{m}^{3}\right)$

$V_{d}=$ Volumen drenado acumulado en el tiempo $t,\left(\mathrm{~m}^{3}\right)$

$\varepsilon=$ Porosidad, $\frac{\mathrm{m}^{3}}{\mathrm{~m}^{3}}$

$x=$ Distancia lineal desde el desagüe, $(\mathrm{m})$

$t=$ Tiempo, (día)

$\beta=$ Pendiente del sistema, $\frac{\mathrm{m}}{\mathrm{m}}$

$L=$ Longitud del lecho, $(\mathrm{m})$

$W=$ Ancho del lecho, $(\mathrm{m})$

$K_{s}=$ Conductividad Hidráulica, $\frac{\mathrm{m}}{\text { día }}$

$h(0,0)=$ Carga hidráulica $x=0$ y $t=0,(\mathrm{~m})$

\section{RESULTADO Y DISCUSIÓN}

Para el afluente y los efluentes de los HCFSSH- $H$. $p$ y los HCFSSH-C. $h$, se presenta un $\mathrm{pH}$ alcalino (Tabla 1 ), la grava aporta alcalinidad en forma de iones carbonato y bicarbonato (Ojeda et al. 2014), además, la edad del relleno (28 años) influye en este parámetro; valores altos de $\mathrm{pH}$ son característicos de rellenos sanitarios de alta edad la edad, mayores de 10 años (Vymazal, 2018; Mosquera \& Lara, 2012). Los valores obtenidos están dentro del rango habitual de $\mathrm{pH}$, en humedales construidos de flujo subsuperficial; las plantas por actividad fotosintética generan un equilibrio buffer y, normalmente, no permiten que el $\mathrm{pH}$ baje y que la actividad de los microorganismos se vea afectada (Montoya et al. 2010).

Los valores medios de oxígeno disuelto obtenidos en los efluentes de los HCFSSH- $H$. $p$ y los HCFSSH-C. $h$ son mayores que los obtenidos para el afluente (Tabla 1). Este leve aumento, se puede deber a que el oxígeno producido por la fotosíntesis en las plantas es liberado desde las raíces al 

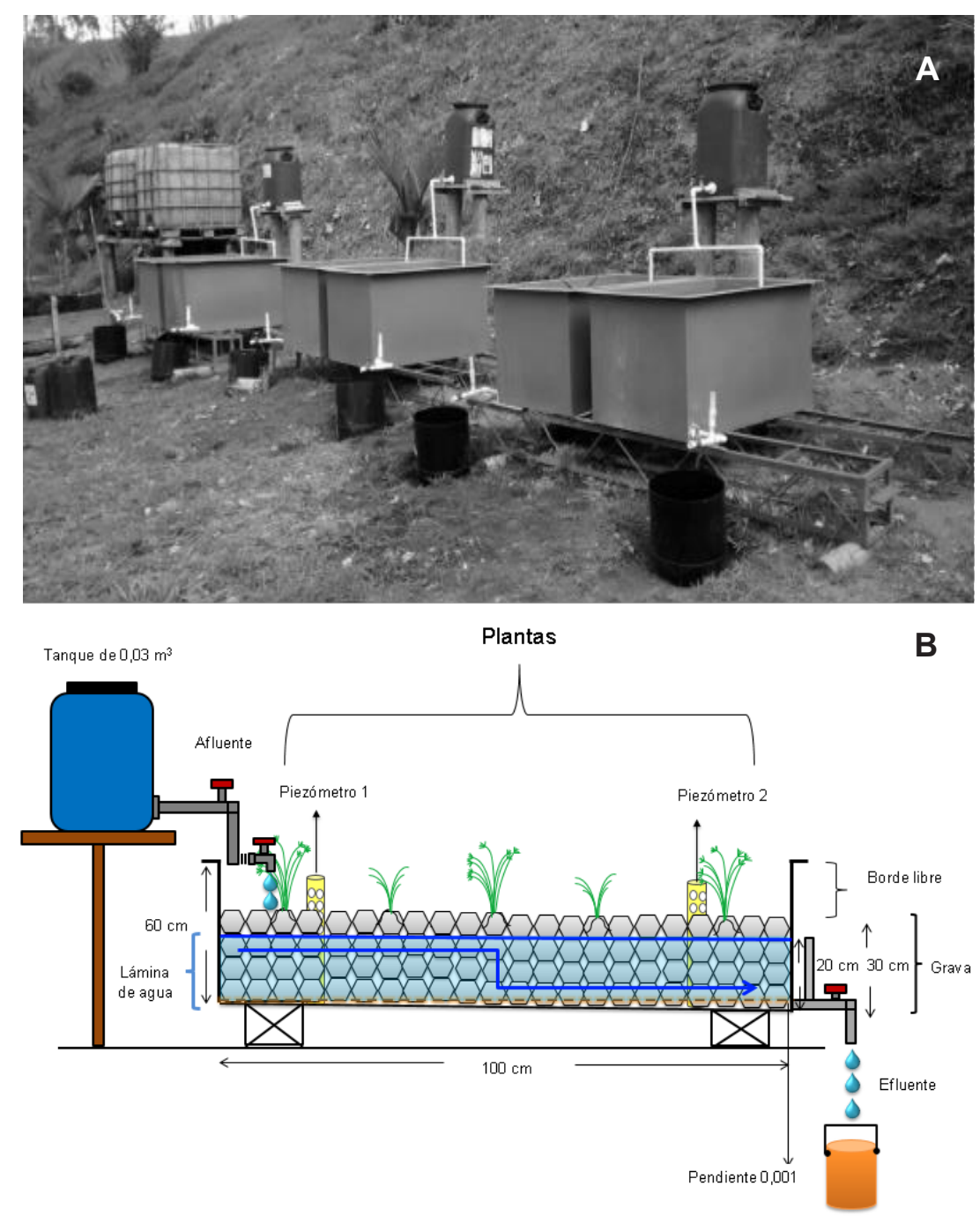

Figura 1. Humedales construidos a escala piloto. A) Ubicación en el relleno sanitario "El Ojito"-Popayán-Cauca y B) Vista en perfil.

Tabla 1. Valores medios de la caracterización fisicoquímica del lixiviado y eficiencias de remoción de los HCFSSH.

\begin{tabular}{|c|c|c|c|c|c|}
\hline \multirow[b]{2}{*}{ Variable } & \multirow[b]{2}{*}{ Afluente } & \multirow{2}{*}{$\begin{array}{c}\text { Efluente } \\
\text { HCFSSH-H. } p\end{array}$} & \multirow{2}{*}{$\begin{array}{c}\text { Efluente } \\
\text { HCFSSH-C. } h\end{array}$} & \multicolumn{2}{|c|}{ Eficiencia de remoción \% } \\
\hline & & & & HCFSSH-H. $p$ & HCFSSH-C. $h$ \\
\hline $\mathrm{Ph}$ & 8,64 & 8,66 & 8,58 & - & - \\
\hline $\mathrm{T}\left({ }^{\circ} \mathrm{C}\right)$ & 21,55 & 20,87 & 21,24 & - & - \\
\hline $\mathrm{CE}(\mu \mathrm{S} / \mathrm{cm})$ & 14820,00 & 5375,83 & 4476,58 & - & - \\
\hline O. D. (mg/L) & 0,35 & 1,01 & 1,26 & - & - \\
\hline DQO (mg/L) & 1494 & 716 & 533 & 51,67 & 64,00 \\
\hline $\mathrm{N}-\mathrm{NO}_{3}{ }^{-}(\mathrm{mg} / \mathrm{L})$ & 123,15 & 68,82 & 62,79 & 45,33 & 48,17 \\
\hline $\mathrm{P}-\mathrm{PO}_{4}{ }^{3-}(\mathrm{mg} / \mathrm{L})$ & 12,33 & 5,02 & 4,05 & 53,67 & 62,67 \\
\hline $\mathrm{Pb}^{2+}(\mathrm{mg} / \mathrm{L})$ & 0,122 & 0,115 & 0,104 & 5,74 & 14,75 \\
\hline
\end{tabular}


medio circundante, influyendo sobre el balance de oxígeno (Austin \& Nivala, 2009; Liu et al. 2016).

La temperatura, tanto en el afluente como en los efluentes de los HCFSSH- $H$. $p$ y de los HCFSSH-C. $h$, no varió significativamente (Tabla 1) y se mantuvo en un rango adecuado para el desarrollo de los procesos biológicos, lo cual, asegura que la cinética al interior de los humedales no sea afectada por temperaturas demasiado bajas (Akratos \& Tsihrintzis, 2007). Los valores en el afluente son ligeramente mayores que en los efluentes; los medios porosos y la vegetación permiten aislar el sistema, manteniendo la temperatura del agua residual tratada (Wang et al. 2017).

Se presentó una disminución significativa de la conductividad eléctrica, tanto en los efluentes de los HCFSSH- $H$. $p$ como los HCFSSH-C. $h$ respecto al afluente (Tabla 1 ); este parámetro muestra el contenido de sólidos y sales disueltas en el agua (Delgadillo et al. 2010), lo que puede indicar una disminución de estas variables en los efluentes de los sistemas, por procesos de sedimentación y de filtración, principalmente

Se aplicó la prueba T pareada (muestras relacionadas), para comparar la eficiencia de remoción de materia orgánica, nutrientes y $\mathrm{Pb}^{+2}$, en los HCFSSH- $H$. $p$ respecto a los $\mathrm{HC}$ FSSH-C. $h$; se evaluó la existencia de diferencias estadísticamente significativas entre las medias de remoción, utilizando una significancia del $5 \%(\alpha=0,05)$, con un nivel de confianza del 95\%. Asimismo, se validó el supuesto de normalidad (prueba de Shapiro-Wilk) en cada una de las variables respuesta, mostrando que los datos provienen de una distribución normal (P-valor $\geq 0,05)$, en todos los parámetros.

DQO: En la figura 2a, se muestran los porcentajes de remoción de $\mathrm{DQO}$, donde se observa que las réplicas de los HCFSSH-H. $p$ como de los HCFSSH-C. $h$ tienen comportamientos similares y la eficiencia fluctúa durante cada muestreo,sin una tendencia clara a aumentar o disminuir en el tiempo; esta dispersión indica que el sistema aún no se ha estabilizado, lo que se puede atribuir a diversos factores, como la variabilidad en la composición del lixiviado (Bulc et al. 1997); la influencia de los procesos metabólicos de los microorganismos en el rendimiento de los humedales (Faulwetter et al. 2009); las variables, como la temperatura, el tiempo de retención hidráulico, la vegetación y el medio de soporte, que influye en la precipitación, la adsorción y en la sinergia entre los procesos fisicoquímicos; la población microbiana y el sustrato (Akratos \& Tsihrintzis, 2007).

El porcentaje de remoción promedio para la $\mathrm{DQO}$ fue mayor en los HCFSSH-C. $h$ que en los HCFSSH- $H$. $p$ (Tabla 1), diferencia que resultó estadísticamente significativa al realizar la prueba $\mathrm{T}$ pareada $(\mathrm{P}$-valor $\leq \mathrm{0,05})$. La transformación de la DQO es esencialmente afectada por los microorganismos; su presencia y su actividad es favorecida por los procesos mediados por las plantas de los humedales (Montoya et al. 2010). Esta diferencia podría indicar que los HCFSSH-C. $h$ presentaron mejores condiciones para la fijación de las comunidades de bacterias degradadoras de la materia orgánica.

Nitratos: En la figura $2 b$, se muestra el comportamiento del porcentaje de remoción de nitratos para los HCFSSH-H. $p$ y los HCFSSH-C. $h$; se puede observar que no hay una tendencia clara a aumentar o disminuir en el tiempo. El porcentaje de remoción promedio fue mayor en los HCFSSH-C. $h$ comparado con los HCFSSH-H. $p$ (Tabla1); sin embargo, esta diferencia no resultó ser estadísticamente significativa (P-valor > 0,05), indicando que la remoción de nitratos está más relacionada con el proceso de desnitrificación que con la asimilación por las plantas. La concentración de nitratos en los humedales construidos aumenta, debido a la nitrificación y disminuye, principalmente, a través de la desnitrificación, proceso que se presenta en condiciones anaeróbicas y representa el $60-95 \%$ de la remoción de nitratos (Spieles \& Mitsch, 1999), La captación de las plantas puede contribuir hasta un 15\% de la remoción de nitrógeno total (Tanner, 2001; Caselles \& García, 2006).

Ortofosfatos: Como se puede observar en la figura 2c, las réplicas de los $\mathrm{HCFSSH}-H$. $p$ como de los HCFSSH-C. $h$ tienen comportamientos similares, presentando una tendencia creciente en la remoción; además, se puede apreciar que las eficiencias obtenidas para los HCFSSH-C. $h$ son mayores que para los $\mathrm{HCFSSH}-H$. $p$. El mayor porcentaje de remoción promedio para $\mathrm{P}_{-} \mathrm{PO}_{4}{ }^{3-}$ se presentó en los HCFSSH-C. $h$ (Tabla 1), mostrando una diferencia estadísticamente significativa (P-valor $\leq 0,05$ ), respecto a los HCFSSH-H. $p$; esto indica, que $C$. haspan presenta una mayor capacidad de asimilación de fósforo que $H$. psittacorum.

El sistema de humedales es nuevo, las plantas aún se encuentran en proceso de crecimiento y el medio de soporte tiene alta capacidad de adsorción; los resultados indican que la remoción de $\mathrm{P}_{-} \mathrm{PO}_{4}{ }^{3-}$ estaría dada por una absorción significativa de las plantas, al igual que la adsorción asociada al medio de soporte limpio.

Plomo: Las eficiencias de remoción de $\mathrm{Pb}^{2+}$ (Figura 2d) fueron afectadas por las bajas concentraciones en el afluente y los efluentes (Tabla 1), obteniendo eficiencias medias, del $5,74 \%$ y $14,75 \%$, para HCFSSH- $H$. $p$ y HCFSSH-C. $h$, respectivamente; sin embargo, el sistema tendió a estabilizarse en las últimas semanas, presentando remociones hasta del 41\%. La capacidad de intercambio catiónico, el pH, la materia orgánica y el nivel de fósforo disponible pueden afectar directamente la capacidad del sustrato para eliminar el $\mathrm{Pb}$; 


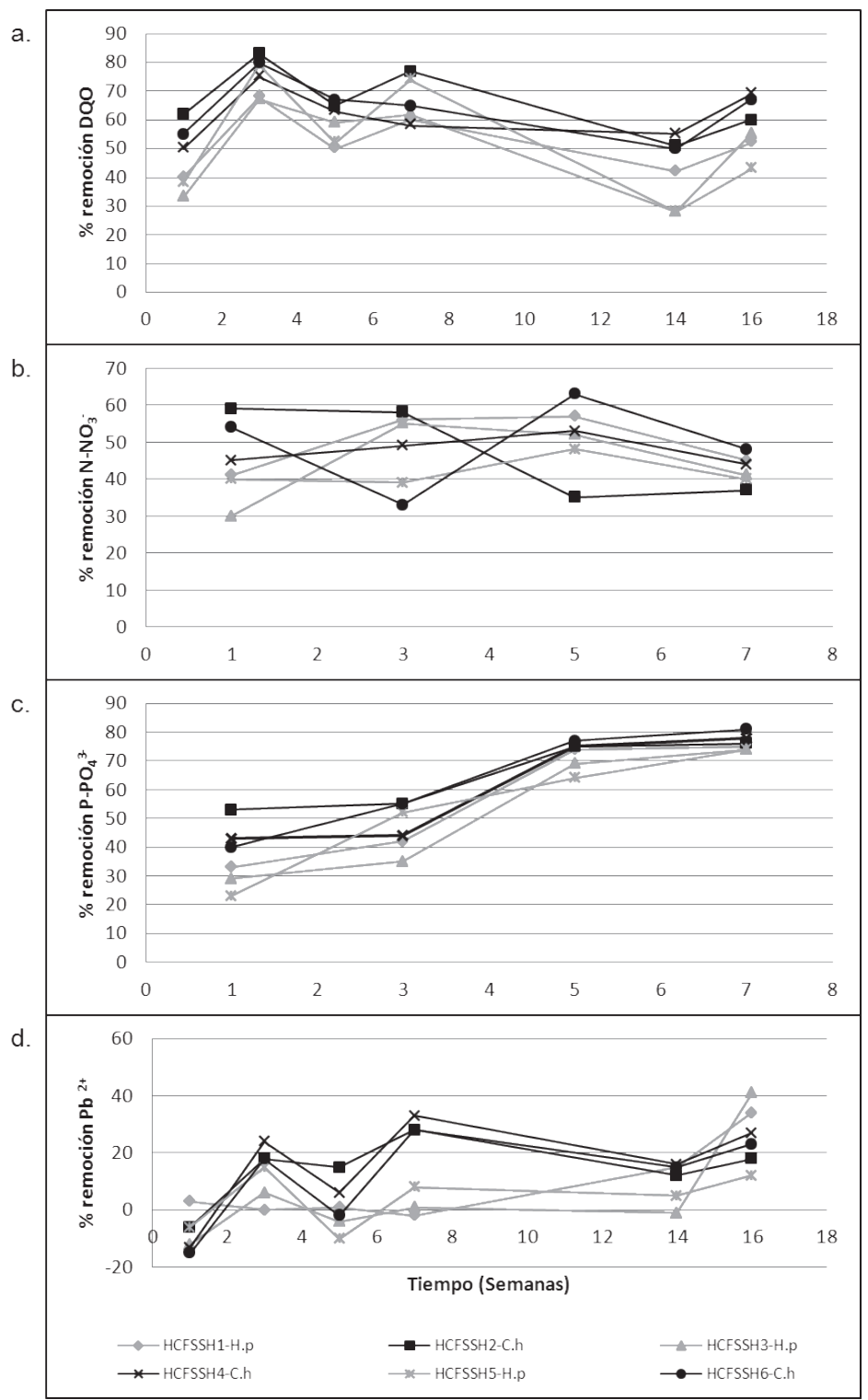

Figura 2. Eficiencias de remoción de los humedales sembrados con Cyperus haspan y Heliconia psittacorum. A) DQO; b) $\mathrm{NO}_{3}^{-}$у с) $\mathrm{PO}_{4}{ }^{3-}$, d. $\mathrm{Pb}^{2+}$.

además, procesos como la disolución de los complejos metal-carbonato y la desorción, liberan el metal al agua, favoreciendo su movilidad, debido, posiblemente, a una caída del potencial de óxido reducción, pH y un déficit de oxígeno, facilitando que otros iones compitan con el metal por llenar espacios (Marchand et al. 2010). Igualmente, los diferentes procesos fisicoquímicos de remoción de metales, conducen a una acumulación en el fondo del humedal, y, por tanto, a la separación de los metales del flujo de agua, si los sedimentos o el sustrato del humedal se remueven puede ocurrir la resuspensión de los metales y, ocasionalmente, su solubilización (Fernández et al. 2004).
Como se puede observar en la figura $2 \mathrm{~d}$, las eficiencias de remoción de $\mathrm{Pb}^{2+}$ obtenidas para los HCFSSH-C. $h$ son mayores que para los HCFSSH-H. $p$, presentando una diferencia estadísticamente significativa (P-valor $\leq 0,05)$, favoreciendo a los HCFSSH-C. $h$; esto sugiere, que $C$. haspan puede presentar mayor capacidad de asimilación de $\mathrm{Pb}^{2+}$ que $H$. psittacorum.

Conductividades hidráulicas: En la tabla 2, se muestran las $K s$, en el P1 y P2, para HCFSSH- $H$. $p$ y HCFSSH-C. $h$; se puede observar que hay variaciones en los valores de entrada y salida, aunque no se presenta una tendencia clara a aumentar o disminuir durante cada muestreo, debido a que 
los sistemas no se han estabilizado, dado el corto tiempo de operación. Los resultados obtenidos por Baptestini et al. (2017), en HCFSSH, con un tiempo de operación similar, indicaron que no fue suficiente para generar una reducción significativa en la Ks; sin embargo, diferentes estudios han mostrado que en los HCFSSH la obstrucción está relacionada, principalmente, por la acumulación y la sedimentación de sólidos de diferente naturaleza y la formación de biopelícula, que llenan lentamente los espacios vacíos del medio de soporte; de igual forma, procesos como la precipitación química, el crecimiento de las raíces y rizomas de las plantas, llevan a un decaimiento de la Ks, a largo plazo y comprometen el tiempo de vida útil de estos sistemas (Vymazal, 2018; Knowles et al. 2010; Ruiz et al. 2010; Pedescoll et al. 2009; Suliman et al. 2006; Tanner \& Sukias, 1995).

Con los datos obtenidos de Ks, en P1 y P2 (Tabla 2), se determinaron parámetros estadísticos (Tabla 3); se observa, que los coeficientes de variación son menores o iguales al 5,36\%, demostrando que se tuvo un buen control en las mediciones, obteniendo observaciones parecidas. De igual forma, se realizó un estudio comparativo entre los HCFSSH- $H$. $p$ y HCFSSH-C. $h$, para los valores medios de Ks, en los P1 y P2, aplicando la prueba no paramétrica de Kruskal-Wallis, la cual, no detectó diferencias estadísticamente significativas ( $p>0,05)$, esto indica, que bajo las condiciones del presente estudio, el tipo de planta no influye sobre este parámetro, a pesar de que $C$. haspan presentó mejor desarrollo. Se evidenció que el crecimiento de las raíces y rizomas de las plantas promueven flujos preferenciales del agua en el fondo del humedal y retie- nen el agua, retrasando su salida y aumentando el tiempo de retención (Bowmer, 1987; Drizo et al. 2000).

Los valores obtenidos de $K s$ en los P2 son menores en comparación a los P1, para todos los sistemas, diferencia que resultó estadísticamente significativa al aplicar la prueba no paramétrica Rangos de Signos de Wilcoxon $(p<0,05)$. Otros estudios en humedales construidos para el tratamiento de aguas residuales han reportado que la zona de entrada es la primera en sufrir disminuciones en este parámetro (Pedescoll et al. 2009; Knowles et al. 2011); sin embargo, los resultados obtenidos, se podrían explicar teniendo en cuenta la variabilidad en la composición del lixiviado, que los sistemas aún no se han estabilizado y la posible resuspensión de los sedimentos o del sustrato, que se van acumulando a la salida de los sistemas (Fernández et al. 2004).

Los sistemas de HCFSSH, sembrados con las plantas nativas tropicales $H$. psittacorum y $C$. haspan, muestran ser una alternativa factible en el tratamiento secundario de lixiviados de rellenos sanitarios maduros para la remoción de $\mathrm{DQO}$,

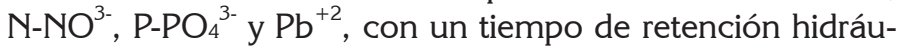
lico de 4 días. C. haspan fue la especie que se adaptó más fácilmente a las condiciones de los sistemas evaluados, posiblemente, por sus características de especie invasiva y su capacidad de desarrollarse en medios inundados, mientras que H. psittacorum presentó síntomas de posibles daños en su fisiología, factores que, seguramente, influyeron en las diferencias de remoción, encontradas para este tipo de plantas.

Tabla 2. Conductividad hidráulica (m/día) en la entrada y salida de los humedales construidos.

\begin{tabular}{|c|c|c|c|c|c|c|}
\hline \multicolumn{7}{|c|}{ Entrada (P1) } \\
\hline \multirow{2}{*}{ MUESTREO } & \multicolumn{6}{|c|}{ HCFSSH } \\
\hline & $1-H . p$ & 2-C. $h$ & 3-H. $p$ & 4-C. h & $5-H . p$ & 6-C. h \\
\hline 1 & 859,68 & 794,88 & 846,72 & 868,32 & 855,36 & 881,28 \\
\hline 2 & 786,24 & 734,40 & 820,80 & 855,36 & 872,64 & 846,72 \\
\hline 3 & 820,80 & 760,32 & 898,56 & 794,88 & 829,44 & 915,84 \\
\hline 4 & 846,72 & 777,60 & 872,64 & 881,28 & 855,36 & 959,04 \\
\hline 5 & 768,96 & 768,96 & 907,20 & 898,56 & 872,64 & 864,00 \\
\hline \multicolumn{7}{|c|}{ Salida (P2) } \\
\hline \multirow{2}{*}{ MUESTREO } & \multicolumn{6}{|c|}{ HCFSSH } \\
\hline & $1-H . p$ & 2-C. $h$ & 3-H. p & 4-C. $h$ & 5-H. p & 6-C. $h$ \\
\hline 1 & 820,80 & 777,60 & 829,44 & 842,40 & 803,52 & 855,36 \\
\hline 2 & 760,32 & 717,12 & 803,52 & 846,72 & 820,80 & 794,88 \\
\hline 3 & 786,24 & 734,40 & 881,28 & 777,60 & 803,52 & 872,64 \\
\hline 4 & 777,60 & 760,32 & 855,36 & 846,72 & 803,52 & 907,20 \\
\hline 5 & 734,40 & 743,04 & 846,72 & 864,00 & 812,16 & 812,16 \\
\hline
\end{tabular}


Tabla 3. Resultados de los parámetros estadísticos de la conductividad hidráulica (m/día) en los P1 y P2.

\begin{tabular}{|c|c|c|c|c|c|c|}
\hline \multicolumn{7}{|c|}{ Entrada (P1) } \\
\hline \multirow{2}{*}{ PARÁMETRO } & \multicolumn{6}{|c|}{ HCFSSH } \\
\hline & $1-H \cdot p$ & 2-C. h & 3-H. $p$ & 4-C. h & $5-H . p$ & 6-C. h \\
\hline Promedio & 816,48 & 767,23 & 869,18 & 859,68 & 857,09 & 893,38 \\
\hline Máximo & 859,68 & 794,88 & 907,20 & 898,56 & 872,64 & 959,04 \\
\hline Mínimo & 768,96 & 734,40 & 820,80 & 794,88 & 829,44 & 846,72 \\
\hline Coeficiente de variación & 4,73 & 2,91 & 4,13 & 4,61 & 2,07 & 5,01 \\
\hline \multicolumn{7}{|c|}{ Salida (P2) } \\
\hline PARÁMETRO & 1-H. p & 2-C. $h$ & 3-H. p & 4-C. $h$ & 5-H. $p$ & 6-C. $h$ \\
\hline Promedio & 775,87 & 746,49 & 843,26 & 835,49 & 808,70 & 848,45 \\
\hline Máximo & 820,80 & 777,60 & 881,28 & 864,00 & 820,80 & 907,20 \\
\hline Mínimo & 734,40 & 717,12 & 803,52 & 777,60 & 803,52 & 794,88 \\
\hline Coeficiente de variación & 4,12 & 3,13 & 3,44 & 4,00 & 0,96 & 5,36 \\
\hline
\end{tabular}

Es de resaltar que este estudio aporta información relevante para el tratamiento de aguas residuales con características similares, permitiendo futuras investigaciones y aplicaciones en diferentes sectores, para su escalamiento, en climas tropicales.

Conflicto de intereses: El manuscrito fue preparado y revisado con la participación de todos los autores, quienes declaramos que no existe ningún conflicto de intereses que ponga en riesgo la validez de los resultados presentados. Financiación: Este estudio fue financiado por la Vicerrectoría de Investigaciones-VRI, de la Universidad del Cauca.

\section{REFERENCIAS}

1. AKINBILE, C.; YUSOFF, M.; AHMAD, Z. 2012. Landfill leachate treatment using sub-surface flow constructed wetland by Cyperus haspan. Waste Management.(USA). 32(7):1387-1393.

2. AKRATOS, C.S.; TSIHRINTZIS, V.A. 2007. Effect of temperature, HRT, vegetation and porous media on removal efficiency of pilot-scale horizontal subsurface flow constructed wetlands. Ecological Engineering. (Paises Bajos). 29(2):173-191.

3. AUSTIN, D.; NIVALA, J. 2009. Energy requirements for nitrification and biological nitrogen removal in engineered wetlands. Ecological Engineering. 35(9):184192.

4. BAPTESTINI, G.C.F.; MATOS, A.T., MARTINEZ, M.A.; 1, BORGES, A.C.; MATOS, M.P. 2017. Hydraulic con- ductivity variability in horizontal subsurface flow constructed wetlands. Eng. Agric. (Brasil). 37(2):333-342.

5. BOWMER, K. 1987. Nutrient removal from effluents by artificial wetland: Influence of rhizosphere aeration and preferential flow studied using bromide and dye tracers. Water Research. (USA). 21(5):591-599.

6. BULC, T.; VRHOVSEK, D.; KUKANJA, V. 1997. The use of constructed wetland for landfill leachate treatment. Water Science and Technology. 35(5):301-306.

7. CASELLES, A.; GARCIA, J. 2006. Performance of experimental horizontal subsurface flow constructed wetlands fed with dissolved or particulate organic matter. Water Reseach. 40(19):3603-3611.

8. CHIEMCHAISRI, C.; CHIEMCHAISRI, W.; JUNSOD, J.; THREEDEACH, S.; WICRANARACHCHI, P. 2009. Leachate treatment and greenhouse gas emission in subsurface horizontal flow constructed wetland. Bioresource Technology. (Países Bajos). 100(16):38083814.

9. CRITES, R.; TCHOBANOGLOUS, G. 1998. Small and Decentralized Wastewater Management Systems. Ed. McGraw-Hill (New York). 1084p.

10. DELGADILLO, C.; CAMACHO, A.; PÉREZ, L.F.; ANDRADE, M. 2010. Depuración de aguas residuales por medio de humedales artificiales. Ed. Nelson Antequera Durán (Bolivia). 115p. 
11. DRIZO, A.; FROST, C.; GRACE, J.; SMITH, K. 2000. Phosphate and ammonium distribution in a pilot-scale constructed wetland with horizontal subsurface flow using shale as a substrate. Water Research. 34(9):2483-2490.

12. FAULWETTER, J.L.; GAGNON, V.; SUNDBER, C.; CHAZARENC, F.; BURR, M.D.; BRISSON, J.; CAMPER, A.N.; STEIN, O.R. 2009. Microbial processes influencing performance of treatment wetlands:A review. Ecological Engineering. 35(6):987-1004.

13. FERNÁNDEZ, J.; BESCOECHEA, E.; MUÑOZ, J.; FERNÁNDEZ, M. 2004. Manual de Fitodepuración. Ed. dentro del Programa Life por el Ayuntamiento de Lorca, la Obra Social de Caja Madrid, la ETSIA de la UPM y la Fundación Global Nature (Eapaña). 32p.

14. FONDER, N.; HEADLEY, T. 2013. The taxonomy of treatment wetlands: a proposed classification and nomenclature system. Ecological Engineering. 51:203-211.

15. KADLEC, R.; WALLACE, D. 2009. Treatment Wetlands. (Second ed.).CRC Press, Boca Raton, FL (New York). 1000p.

16. KNOWLES, P.; DOTRO, G.; NIVALA, J.; GARCÍA, J. 2011.Clogging in subsurface-flow treatment wetlands: occurrence and contributing factors. Ecological Engineering. 37(2):99-112.

17. KNOWLES, R.P.; GRIFFIN, P.; DAVIES, A. 2010. Complementary methods to investigate the development of clogging within a horizontal sub-surface flow tertiary treatment wetland. Water Research. 44(1):320-330.

18. LIU, H.; HU, Z.; ZHANG, J.; NGO, H.; GUO, W.; LIANG, S.; FAN, J.; LU, S.; WU, H. 2016. Optimizations on supply and distribution of dissolved oxygen in constructed wetlands: A review. Ecological Engineering. 214:797-805.

19. MADERA, P. 2016. Tratamiento de lixiviados de relleno sanitario por medio de humedalesconstruidos sembrados con policultivos de plantas nativas. Ingeniería y Competitividad. (Colombia). 18(2):183-192.

20. MARCHAND, L.; MENCH, M.; JACOB, D.L.; OTTE, M.L. 2010. Metal and metalloid removal in constructed wetlands, with emphasis on the importance of plants and standardized measurements: A review. Environmental Pollution. (Reino Unido). 158(12):3447-3461.
21. MONTOYA, J.; CEBALLOS, L.; CASAS, J.; MORATÓ, J. 2010. Estudio comparativo de la remoción de materia orgánica en HCFSSH usando tres especies de macrófitas. Revista EIA (Colombia). 7(14):75-84.

22. MOSQUERA, J.; LARA, J. 2012. Tratamiento de lixiviados mediante humedales artificiales: revisión del estado del arte. Revista Tumbaga. (Colombia).1(7):73-99.

23. NIVALA, A.; KNOWLES, P.; DOTRO, G.; GARCÍA, J.; WALLACE, S. 2012. Clogging in subsurface-flow treatment wetlands: measurement, modeling and management. Water Research.46(6):1625-1640.

24. OJEDA, L.; ROMERO, S.; REYES, J.; GALLEGOS, R.; HERRERA, A. 2014. Evaluación de la depuración de agua gris en un humedal artificial a escala piloto. En: Rivas, A.; PAREDES, D. (eds). Sistemas de humedales para elmanejo, tratamiento y mejoramiento de la calidad del agua. Ed. Instituto Mexicano de Tecnología del Agua (México). p.66-69.

25. PEDESCOLL, A.; UGGETTI, E.; LLORENS, E.; GRANÉS, F.; GARCÍA, D.; GARCÍA, J. 2009. Practical method based on saturated hydraulic conductivity used to asses clogging in subsurface flow constructed wetlands. Ecological Engineering. 35(8):1216-1224.

26. RENOU, S.; GIVAUDAN, G.; POULAIN, S.; DIRASSOUYAN, F.; MOULIN, P. 2008. Landfill leachate treatment: Review and opportunity. Journal of Hazardous Materials. (Paises Bajos).150(3):468-493.

27. RUIZ, I.; DÍAZ, M.A.; CRUJUIRAS, B.; SOTO, M. 2010. Solids hydrolysis and accumulation in a hybrid anaerobic digester-constructed wetlands system. Ecological Engineering. 36(8):1007-1016.

28. SANFORD, W.; STEENHUIS, T.; PARLANGE, J.; SURFACE, J.; PEVERLY, J. 1995. Hydraulic conductivity of gravel and sand as substrates in rock-reed filters. Ecological Engineering. 4(4):331-336.

29. SPEER, S.; CHAMPAGNE, P.; ANDERSON, B. 2012. Pilot-scale Comparison of two hybrid-passive landfill leachate treatment systems operated in a cold climate. Bioresource Technology. 104:119-126.

30. SPIELES, D.J.; MITSCH, W.J. 1999. The effects of season and hydrologic and chemical loading on nitrate retention in constructed wetlands: a comparison of low-and high-nutrient riverine systems. Ecological Engineering. 14(1/2):77-91. 
31. SULIMAN, F.; FRENCH, H.K.; HAUGEN, L.E.; SØVIKB, A.K. 2006. Change in flow and transport patterns in horizontal subsurface flow constructed wetlands as a result of biological growth. Ecological Engineering. 27(2):124-133.

32. TANNER, C. 2001. Plants as ecosystem engineers in subsurface-flow treatment wetlands Water Science and Technology. (Reino Unido). 44(11/12):9-17.

33. TANNER, C.; SUKIAS, J.P. 1995. Accumulation of organic solids in gravel bed constructed wetlands. Water Science and Technology. 32(3):229-239.

34. VYMAZAL, J. 2014. Constructed wetlands for treatment of industrial wastewaters: A review. Ecological Engineering. 73:724-751.

35. VYMAZAL, J. 2018. Does clogging affect long-term removal of organics and suspended solids in gravel-based horizontal subsurface flow constructed wetlands? Chemical Engineering Journal. (Paises Bajos). 100(16):3808-3814.
36. WALLACE, J.; CHAMPAGNE, P.; MONNIER, A. 2015. Performance evaluation of a hybrid-passive landfill leachate treatment system using. Waste Management. 35:159-169.

37. WANG, M.; ZHANG, D.; DONG, J.; TAN, S. 2017. Constructed wetlands for wastewater treatment in cold climate - A review. Journal of Environmental Sciences. (Paises Bajos). 57:293-311.

38. WOJCIECHOWSKA, E.; GAJAWSKA, M.; OBARSKA, H. 2010. Treatment of landfill leachate by constructed wetlands: there case studies. Polish Journal of Environmental Studies. (Polonia).19(3):643-650.

39. YALCUK, A.; UGURLU, A. 2009. Comparison of horizontal and vertical constructed wetland systems for landfill leachate treatment. Bioresource Technology. 100(9):2521-2526.

Recibido: Noviembre 20 de 2017

Aceptado: Septiembre 8 de 2018

Cómo citar:

Jiménez-Cerón, Y.F.; Delgado-Calvache, L.I.; Fernández-Tulande, C.; Pino-Alegría, H.M.; Casas-Zapata, J.C.; Madera-Parra, C.A.; Lara-Borrero, J.A.; Morató-Farreras, J.; Rengifo-Canizales, E. 2018. Tratamiento de lixiviados utilizando humedales construidos y determinación de conductividades hidráulicas en clima tropical. Rev. U.D.C.A Act. \& Div. Cient. 21(2):543-552. https://doi.org/10.31910/rudca.v21.n2.2018.979 